\title{
NOVEDADES PARA LA FLORA VASCULAR DE LA PROVINCIA DE SANTA FE (ARGENTINA)
}

\author{
Exner, E. ${ }^{1}$; Cerino, C. ${ }^{1}$; Kern, V. ${ }^{1}$; Richard, G. ${ }^{1}$;

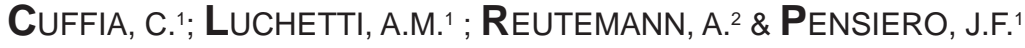

\begin{abstract}
RESUMEN
Producto de viajes de colección realizados en la provincia de Santa Fe, se documentaron 13 taxones novedosos para la provincia, 10 son taxones nativos y 3 son introducidos, siendo uno de éstos últimos una nueva cita, para Argentina.

Palabras clave: Flora, Santa Fe, novedades taxonómicas.
\end{abstract}

\begin{abstract}
Novelties for the vascular flora of Santa Fe province (Argentina).

As a result of collection trips carried out in the province of Santa Fe, 13 taxa were documented for the first time for the province, 10 native and 3 introduced taxa, one of the latter representing a new report for Argentina.

Key words: Flora, Santa Fe, novelties taxonomic.
\end{abstract}

\footnotetext{
1.- Cátedra de Botánica Sistemática Agronómica. Facultad de Ciencias Agrarias, Universidad Nacional del Litoral. Kreder 2805. 3080HOF, Esperanza, Santa Fe, Argentina. Email: eexner@fca.unl.edu.ar

2.- Cátedra de Morfología Vegetal, Facultad de Ciencias Agrarias, Universidad Nacional del Litoral. Kreder 2805. 3080HOF, Esperanza, Santa Fe, Argentina.

Manuscrito recibido el 13 de abril de 2020 y aceptado para su publicación el 30 de junio de 2020.
}

Exner, E.; Cerino, C.; Kern, V.; Richard, G.; Cuffia, C.; Luchetti, A.M.; Reuteman, A.; Pensiero, J.F.

Novedades para la flora vascular de la provincia de Santa Fe (Argentina).

FAVE - Ciencias Agrarias 20 (1): 47-54. CC BY-NC-SA 4.0 


\section{INTRODUCCIÓN}

La flora de la provincia de Santa Fe ha sido estudiada parcialmente por distintos autores a través del tiempo (Brumnich y Marchetti, 2014; Exner et al., 2004, 2013; García, 1993; Luchetti, 1993, 2001, 2009, 2014; Luchetti y Exner, 2018; Luchetti et al., 2009; Marino y Pensiero, 2006; Marino et al., 2006; Pensiero, 1988; Pensiero y Carletti, 1996; Pensiero y de la Peña, 1999; Pensiero y Zabala, 2017; Ragonese, 1940; Vegetti, 1988, 1994). El primer trabajo de conjunto sobre la flora vascular de la provincia (Pensiero et al., 2005), cita para Santa Fe un total de 1969 taxones, e incluye claves para el reconocimiento de las familias y géneros.

Producto de viajes realizados en la provincia de Santa Fe en los últimos diez años en el marco del Programa de Documentación, Conservación y Valoración de la flora nativa (PRODOCOVA), programa declarado de Interés Institucional por la Universidad Nacional del Litoral, se efectuaron numerosas colectas de la flora regional. El estudio de estas colecciones ha revelado la presencia de nuevas citas florísticas para la provincia de Santa Fe. Por lo dicho, el objetivo de este trabajo es dar a conocer los nuevos taxones hallados para esta provincia, uno de los cuales resulta, además, nueva cita para Argentina.

\section{MATERIALES Y MÉTODOS}

Se realizaron distintos viajes al centro y norte de la provincia de Santa Fe (especialmente a los departamentos Vera, General Obligado, San Javier, Las Colonias y La Capital), con el objetivo de documentar la flora regional. En cada ambiente visitado se realizaron colectas de especímenes de la flora presente, siguiendo las especificaciones indicadas por Katinas (2001). Dichos especímenes, luego de disecados y acondicionados, se conservaron en el Herbario "Arturo E. Ragonese" (SF) de la Facultad de Ciencias Agrarias de la Universidad Nacional del Litoral. Imágenes fotográficas que permiten ilustrar distintos aspectos de los taxones citados, como su hábito, inflorescencia, flores y/o frutos se incluyeron en el Banco de imágenes IRUPÉ... (2020). Se ha considerado nueva cita para la provincia cuando los taxones no fueron mencionados previamente para Santa Fe por ninguno de los trabajos florísticos señalados, además de no citarse esta provincia en la distribución que se señala para cada taxón en Flora Argentina... (2018), ni en la actualización del catálogo de plantas vasculares del Cono Sur (Zuloaga et al., 2019).

Los taxones se citan alfabéticamente, y para cada uno se indica: a) su nombre científico aceptado y su familia botánica, siguiendo la nomenclatura propuesta en Flora Argentina... (2018), b) la bibliografía botánica que permite su determinación taxonómica, c) distribución y hábitat, d) el material estudiado (ejemplar de referencia) y e) observaciones, cuando corresponde. 
Para las especies introducidas (exóticas, no nativas), se tuvieron en cuenta las categorías propuestas por Richardson et al. (2011) quienes reconocen a lo largo del continuum del proceso de invasión las categorías de taxón introducido casual, naturalizado e invasor.

\section{RESULTADOS}

Se incluye un total de 13 taxones, de los cuales 10 corresponden a taxones nativos de Argentina y los otros 3 a taxones introducidos, uno de estos últimos resulta ser, además, nueva cita para la flora de Argentina.

Canavalia bonariensis Lindl. (Fabaceae) Referencias bibliográficas. Burkart, 1987; Sauer, 1964; Ulibarri et al., 2002.

Distribución y hábitat. Especie nativa de Argentina, Brasil, Paraguay y Uruguay. En Argentina crece en Buenos Aires, Chaco, Corrientes, Entre Ríos, Jujuy y Santa Fe. Típica de selvas húmedas, marginales, a lo largo del río Uruguay y del Paraná y margen sur del Plata.

Material estudiado. Prov. Santa Fe. Dpto. Las Colonias, María Luisa, banquina de camino rural a $500 \mathrm{~m}$ de RP 62, 03-VII2019, Exner E. 1039 (SF).

Observación. Se la ha observado en la localidad de Sauce Viejo del departamento La Capital, pero no se cuenta con ejemplar de herbario.

Commelina platyphylla Klotzch ex Seub. (Commelinaceae)

Referencias bibliográficas. Bacigalupo, 1964; Clarke, 1881.

Distribución. Especie nativa de Argentina, Brasil, Paraguay y Uruguay. En Argen- tina crece en Chaco, Corrientes, Entre Ríos, Formosa, Misiones y Santa Fe.

Material estudiado. Prov. Santa Fe. Dpto. Vera, 2 km al Este de Los Tábanos, $28^{\circ} 27^{\prime} 40,1^{\prime \prime} \mathrm{S}$ 5958'19,1” W, 56 m s.m., 21-II-2018, Pensiero J. et al. 13016 (SF).

Cyperus rigens J. Presl var. impolitus (Kunth) Hefler \& Longhi-Wagner (Cyperaceae)

Referencias bibliográficas. Hefler, 2010; Pedersen, 1972.

Distribución. Variedad nativa de Argentina, Brasil, Paraguay y Uruguay. En Argentina crece en las provincias de Buenos Aires, Chaco, Córdoba, Corrientes, Entre Ríos, Formosa, Misiones y Santa Fe.

Material estudiado. Prov. Santa Fe. Dpto. General Obligado, Ruta 100-S, 5 km de la RN 11 camino a Villa Guillermina, en un bosque, 08-XII-1986, Vegetti 758 (SF).

Dioscorea campestris Griseb. (Dioscoreaceae)

Referencias bibliográficas. Hauman, 1916; Hurrell et al., 2009; Xifreda, 1990.

Distribución y hábitat. Especie nativa de Argentina, Brasil, Paraguay y Uruguay. En Argentina crece en Chaco, Corrientes, Entre Ríos, Formosa, Misiones, Salta, Tucumán y Santa Fe. Habita en bosques, matorrales y campos secos.

Material estudiado. Prov. Santa Fe. Dpto. Vera, $2 \mathrm{~km}$ al Este de Los Tábanos, $28^{\circ} 27^{\prime} 40,1^{\prime \prime} \mathrm{S} 59^{\circ} 58^{\prime} 19,1^{\prime \prime} \mathrm{W}, 56 \mathrm{~m}$ s.m., 21-II-2018, Pensiero J. et al. 13012 (SF).

Erechtites valerianifolius (Link ex Spreng.) Less. ex DC. f. valerianifolius (Asteraceae)

Referencias bibliográficas. Cabrera, 1974; Freire, 2014. 
Distribución. Forma nativa de Argentina, Brasil, Paraguay y Uruguay. En Argentina crece en las provincias de Chaco, Corrientes, Entre Ríos, Formosa, Misiones, Salta y Santa Fe.

Material estudiado. Prov. Santa Fe. Dpto. General Obligado, $5 \mathrm{~km}$ al E del Km 844 de la RN 11, establecimiento "Guapoí", 2842' 58,3”'S 59²3’07,7” W, 51 m s.m., 30-X-2016, Pensiero J. et al. 11358 (SF).

Evolvulus sericeus Sw. f. pedunculatus Ooststr. (Convolvulaceae)

Referencias bibliográficas. Buck, 1979; Chiarini y Anton, 2019; Chiarini y Ariza Espinar, 2006.

Distribución. Forma nativa de Argentina, Estados Unidos y México. En Argentina crece en las provincias de Entre Ríos, Formosa, Jujuy y Santa Fe.

Observación. Chiarini \& Anton (2019) consideran que este taxón es dudoso para la flora de Argentina, aunque el material que hemos estudiado concuerda con su descripción.

Material estudiado. Prov. Santa Fe. Dpto. Vera, RN 11, salitral de Berna, próximo a la localidad de Berna, 29¹7'03,6" S 5950'51,4" W, 17-XII-2012, Pensiero J. et al. 8990 (SF).

Hydrocotyle cryptocarpa Speg. (Araliaceae)

Referencias bibliográficas. Pérez - Moreau,1938; Pontiroli, 1966; Zuloaga, 2019.

Distribución y hábitat. Especie nativa de Argentina, Chile y Uruguay. En Argentina crece en las provincias de Buenos Aires, Corrientes, Entre Ríos y Santa Fe. Habita en charcos y lagunas temporarias sobre tierras arcillosas e impermeables. Germina desde fines del invierno en charcos, florece y fructifica en el período de desecación, desaparece a principios de verano.

Material estudiado. Prov. Santa Fe. Dpto. Vera, Las Gamas, 29॰27'16,5"S 60²3'12,3” W, 59 m s.m., 14-XI-2017, Pensiero J. et al. 12412 (SF).

Nothoscordum bonariense (Pers.) Beauverd (Amaryllidaceae)

Referencias bibliográficas. Guaglianoni,1972; Sassone et al., 2014.

Distribución y hábitat. Especie nativa de Argentina, Brasil y Uruguay. En Argentina crece en las provincias de Buenos Aires, Corrientes, Entre Ríos, Misiones y Santa Fe. Habita lugares húmedos del centro-norte de la provincia.

Material estudiado. Prov. Santa Fe. Dpto. Vera, Las Gamas, 29²6’21,9”S $60^{\circ} 23^{\prime} 17,1$ ”W, 25-IX-2019, Exner E. et al. 1132 (SF).

Rhipsalis cruciforme (Vell.) A. Cast. (Cactaceae)

Referencias bibliográficas. Barthlott y Taylor, 1995; Taylor, 1994.

Distribución. Especie nativa de Argentina, Brasil, Paraguay y Uruguay. En Argentina crece en Chaco, Corrientes, Formosa, Misiones y Santa Fe.

Material estudiado. Prov. Santa Fe. Dpto. General Obligado, RN 11, 6 km al E del km 844, establecimiento "Guapoí", $28^{\circ} 42^{\prime} 54,8^{\prime \prime} \mathrm{S} 59^{\circ} 23^{\prime} 13,7^{\prime \prime} \mathrm{W}, 32$ m s.m., 13-VIII-2019, Pensiero J. y J. Zabala 13809 (SF). 
Rhynchospora tenuis Link subsp. tenuis (Cyperaceae)

Referencias bibliográficas. Koyama, 1972; Thomas, 1992.

Distribución. Subespecie nativa, que crece desde Méjico, llegando a Argentina, Brasil, Paraguay y Uruguay. En Argentina crece en Corrientes, Entre Ríos, Jujuy, Misiones y Santa Fe.

Material estudiado. Prov. Santa Fe. Dpto. La Capital, Colastiné, en lote de la zona urbana, 28-II-2018, Reutemann A. et al. 198 (SF).

Sagina apetala Ard. var. apetala (Caryophyllaceae)

Referencias bibliográficas. Añón, 1967; Martínez Crovetto,1967; Pedersen, 1987.

Distribución y hábitat. Variedad originaria de Europa occidental y norte de África. Especie introducida (casual) en Argentina, Chile, Paraguay y Uruguay. En Argentina crece en Buenos Aires, Corrientes, Entre Ríos y Santa Fe. Crece en terrenos bajos, inundables, suelos arcillosos.

Material estudiado. Prov. Santa Fe. Dpto. Vera, Las Gamas, 29²6'19,3's $60^{\circ} 22^{\prime} 51,8^{\prime \prime} \mathrm{W}, 04-\mathrm{IX}-2019$, Exner et al. 1109 (SF).

Scandix pecten-veneris L. (Apiaceae) Referencias bibliográficas. Pedersen, 2005.

Distribución y hábitat. Especie originaria de Europa, Asia menor y África. Especie introducida (casual) en Argentina y en Chile. En Argentina crece en Entre Ríos y Santa Fe.

Observación. Para la flora europea recibe el nombre de "peine de Venus" o "agujas del pastor".
Material estudiado. Prov. Santa Fe. Dpto. La Capital, Santa Fe, Reserva Urbana del

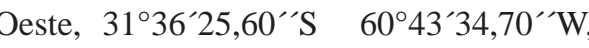
15 m s.m., 25-X-2018, Pensiero J. et al. 13114 (SF).

Scrophularia peregrina L. (Scrophulariaceae)

Referencias bibliográficas. Tutin et al., 1972; Uzunhisarcikli et al., 2018.

Distribución y hábitat. Especie originaria del Sur de Europa, Norte de África y Suroeste de Asia. Especie introducida (casual) en Argentina, donde crece en Santa Fe escapada de cultivo (observación de los autores). Naturalmente habita en lugares sombríos y húmedos.

Observación. Si bien se trata de una especie que puede cultivarse como ornamental en el presente trabajo se documenta su presencia creciendo sin la intervención del hombre presentándose como maleza en jardín urbano. Tratándose de esta forma en nueva cita para Argentina.

Material estudiado. Prov. Santa Fe. Dpto. Las Colonias, Esperanza, en la ciudad, 10-X-2013, Exner E. 107 (SF).

\section{BIBLIOGRAFÍA}

Añón D. 1967. Caryophyllaceae. En: Cabrera AL, editor. Fl. Prov. Buenos Aires, Colecc. Ci. Inst. Nac. Tecnol. Agropecu. 4(3 $\left.3^{\mathrm{a}}\right)$ :192-237.

Bacigalupo NM. 1964. Estudio sobre Commelinaceae argentinas I. Darwiniana 13(1): 87-103.

Barthlott WA, Taylor NP. 1995. Notes toward a monograph of Rhipsalidae (Cactaceae). Bradleya 13:43-79. 
Banco de imágenes IRUPE. 2020. Esperanza, Facultad de Ciencias Agrarias-Universidad Nacional del Litoral. Disponible en https:// www.fca.unl.edu.ar/prodocova/IRUPE. [Acceso 29 de junio 2020].

Brumnich F, Marchetti ZY. 2014. Rhabdocaulon strictus (Benth.) Epling (Lamiaceae) en humedales del Río Parana: Nueva cita para la flora de Santa Fe, Argentina. Scientia Interfluvius 5(2):78-85.

Buck HT. 1979. Convolvulaceae. En: Burkart A, editor. Fl. Il. Entre Ríos, Colecc. Ci. Inst. Nac. Tecnol. Agropecu. 6 (5):148-194.

Burkart A. 1987. Leguminosae. En: Troncoso NS y Bacigalupo NM, editores. Flora Ilustrada de Entre Ríos (Argentina). Colecc. Ci. Inst. Nac. Tecnol. Agropecu. 6 (3a): 442-738.

Cabrera AL. 1974. Compositae. En: Burkart A, editor. Fl.Il. Entre Ríos (Argentina). Colecc. Ci. Inst. Nac. Tecnol. Agropecu 6 (6): 106-554.

Chiarini F, Ariza Espinar L. 2006. Convolvulaceae. Fl. Fanerog. Argent. 96:1-81.

Chiarini F, Anton AM. 2019. Familia Convolvulaceae. Flora Argentina-Flora vascular de la República Argentina 20 (2): 269-380.

Clarke CB. 1881. Commelinaceae. Monog. Phaner. 3: 112-324.

Exner E, D’Angelo C, Pensiero JF. 2004.Vegetación y flora de la reserva universitaria de la Escuela Granja de Esperanza (Santa Fe, Argentina). Revista FAVE, Ciencias Agrarias 3 (1-2): 53-76.

Exner E, Luchetti AM, Kern V, Gutierrez HF, Cerino MC, Marinoni L, Richard G, Zabala JM, Bortoluzzi A, D’Angelo C y Pensiero JF. 2013. Novedades para la flora vascular de la provincia de Santa Fe. XXXIV Jornadas Argentinas de Botánica. Bol. Soc. Argent. Bot. Vol. 48 (Suplemento) pg. 257.

Flora Argentina. 2018. Buenos Aires: Instituto de Botánica Darwinion. Disponible en http://www. floraargentina.edu.ar [Acceso 29 de junio de 2020].
Freire S. 2014. Dicotyledoneae-Asteraceae (Senecioneae a Vernonieae). En Zuloaga F, Belgrano M, Anton A, editores. Flora vascular de la República Argentina 7(3) p. Instituto de Botánica Darwinion.

García RC. 1993. Flora Argentina I. Las Tropaeolaceae de la provincia de Santa Fe. II. Las Bignoniaceae de la provincia de Santa Fe. Servicio de Publicaciones Universidad Nacional de Rosario. 33 pp.

Guaglianoni ER. 1972. Nueva circunscripción de la Tribu Leucocoryneae (Amaryllidaceae, Allioideae). Darwiniana 17:159-240.

Hauman LL. 1916. Les Dioscoréacées de l' Argentine. Anales Mus. Nac. Hist. Nat. Buenos Aires 27: 441-513.

Hefler SM. 2010. Novidades taxonômicas em espécies de Cyperus subg. Cyperus (Cyperaceae). Rodriguésia. 61 (Supl.): 07-14.

Hurrell JA, Delucci G, Correa MN, Sánchez MI, Roitman G, Constantino FB, Ulibarri EA, Guaglianone ER,Tur NM. 2009. Flora rioplatense: sistemática, ecología y etnobotánica de las plantas vasculares rioplatenses. LOLA. III (4): pg.

Katinas L. 2001. El Herbario. Significado, Valor y Uso. PROBIOTA. Serie Técnica y Didáctica 1. 11 pp.

Koyama TM. 1972. Rhynchosporaea and Cladieae. Mem. New York Bot. Gard.23:23-89.

Luchetti AM. 1993. Los Géneros de Solanáceas de la Provincia de Santa Fe. Revista FAVE 7 (2):38-47.

Luchetti AM. 2001. Las Solanáceas de la provincia de Santa Fe. Natura Neotropicalis 32(2):111-132.

Luchetti AM. 2009. Claves para el reconocimiento de las especies santafesinas de las Leguminosas papilionoideas. Natura Neotropicalis 39 (1y2): 47-81.

Luchetti AM. 2014. Las Leguminosas Mimosoideas de la provincia de Santa Fe. Natura Neotropicalis 44(1-2): 21-43. 
Luchetti AM, Exner E. 2018. Las Leguminosas Cesalpinioideas de la provincia de Santa Fe. Revista FAVE Sección Ciencias Agrarias 17(2):39-52.

Luchetti AM, Kern VG, Cerino C y Pensiero J F. 2009. Nuevas citas para la provincia de Santa Fe, Argentina. XXXII Jornadas Argentinas de Botánica. Bol. Soc. Argent. Bot. Vol. 44 (Suplemento) pg. 92.

Marino GD, Pensiero JF. 2006. Catálogo de árboles y arbustos de la provincia de Santa Fe. Com. Museo Provincial Ciencias Naturales “Florentino Ameghino" 12(1):1-28.

Marino GD, Marchetti ZY, Pensiero JF. 2006. Clave para el reconocimiento de la flora leñosa nativa del Sitio Ramsar Jaaukanigás (Provincia de Santa Fe, Argentina). Bol. Soc. Argent. Bot. 41(1-2):77-84.

Martínez Crovetto R. 1967. Catálogo preliminar de las Caryophyllaceae de la Argentina y del Uruguay. Bonplandia 2:187-264.

Pedersen TM. 1972. Cyperus laetus Presl and Cyperus rigens Presl, two badly understood South American sedges, with notes on some related species. Darwiniana 17:527-547.

Pedersen TM. 1987. Caryophyllaceae. En: Troncoso De Burkart NS, Bacigalupo NM editores. Fl.Il. Entre Ríos, Colecc. INTA. 6 (3): 251-291.

Pedersen TM. 2005. Umbelíferas. En: Bacigalupo, NM, editora. Fl. Il. Entre Ríos, Colecc. Ci. Inst. Nac. Tecnol. Agropecu. 6:4b: 554-598.

Pensiero JF. 1988. Los géneros de Poaceae de la Provincia de Santa Fe. Revista Facultad de Agronomía y Veterinaria -UNL- 3(1 2):1 22.

Pensiero JF, Carletti PD. 1996. Catálogo de las gramíneas de la provincia de Santa Fe. XXV Jornadas Argentinas de Botánica, Mendoza.

Pensiero JF, de la Peña MR. 1999. Flora y Avifauna de la provincia de Santa Fe. Talleres gráficos El Litoral Argentino, 384 pp.
Pensiero JF, Zabala JM. 2017. Recursos fitogentéticos forrajeros nativos y naturalizados para los Bajos Submeridionales: prospección y priorización de especies para planes de introducción a cultivo. Revista FAVE Sección Ciencias Agrarias. 16(1):67-98.

Pensiero JF, Gutiérrez HF, Luchetti AM, Exner E, Kern V, Brnich E, Oakley l, Prado D, Lewis JP. 2005. Flora vascular de la provincia de Santa Fe. Claves para el reconocimiento de las familias y géneros. Catálogo sistemático de las especies. 403 pp.

Pérez -Moreau, R.A. 1938. Revisión de las Hydrocotyle argentinas. Lilloa 2:413-463.

Pontiroli, A. 1966. Umbelliferae. En: Cabrera AL, editor. Flora de la provincia de Buenos Aires. Colecc. Ci. Inst. Nac. Tecnol. Agropecu. 4 (4): 337-403.

Ragonese AE. 1940. La flora halófila del sur de la provincia de Santa Fe (R.A.). Darwiniana 7:401.

Richardson DM, Pyšek P, Carlton JC. 2011. A compendium of essential concepts and terminology in biological invasions. En: Richardson DM, editor. Fifty years of invasion ecology: the legacy of Charles Elton, pp. 409420. Blackwell Publ., Oxford.

Sassone AB, Arroyo-Leuenberger SC, Giussani LM. 2014. Nueva circunscripción de la Tribu Leucocoryneae (Amaryllidaceae, Allioideae). Darwiniana n.s. 2:197-206.

Sauer J D.1964. Revision of Canavalia. Brittonia 16:663-796.

Taylor N. 1994. Proposal to conserve Cactus cruciformis Vell. (Cactaceae). Taxon 43:469-470.

Thomas WW. 1992. A synopsis of Rhynchospora (Cyperaceae) in Mesoamerica. Brittonia 44: 14-43.

Tutin TG y otros. (eds.). 1972. Flora Europaea 3: 1-370. Cambridge University Press. 
Ulibarri EA, Gomez Sosa EV, Cialdella AM, Vegetti AC. 1994. Notas sobre Cyperaceae de Fortunato RH, Bazzano D. 2002. Leguminosas nativas y exóticas. En: Hurrell JA, Lahitte HB editores. Biota Rioplatense VII. L.O.L.A. Buenos Aires, 320 p. la provincia de Santa Fe. II. Los géneros de Cyperaceae de la provincia de Santa Fe. Rev. FAVE 7(2):31-37.

Xifreda CC. 1990. Sobre la presencia de una "vaina cristalífera” en el tallo de algunas especies de Dioscorea (Dioscoreaceae). Darwiniana 30:137-142.

Zuloaga FO, Belgrano M, Zanotti CA. 2019. Actualización del catálogo de las planas vasculares del Cono Sur. Darwiniana, nueva serie $7(2): 208-278$. maleza que se está difundiendo en el norte de la provincia. Rev. FAVE 3(1-2):23-24. 